\title{
H.E.S.S. and Fermi-LAT Observations of PSR B1259-63/LS 2883
}

Thomas Murach*

DESY, D-15738 Zeuthen, Germany

E-mail: thomas . murach@desy. de

\section{Pol Bordas}

Institut de Ciències del Cosmos (ICC UB), Universitat de Barcelona (IEEC-UB), Martí

Franquès 1, E08028 Barcelona, Spain

E-mail: pbordasefqa . ub . edu

\section{Christian Mariaud}

Laboratoire Leprince-Ringuet, École Polytechnique, UMR 7638, CNRS/IN2P3, Institut Polytechnique de Paris, F-91128 Palaiseau, France

E-mail: mariaudellr.in2p3.fr

\section{Carlo Romoli}

Max-Planck-Institut für Kernphysik, P.O. Box 103980, D 69029 Heidelberg, Germany

E-mail: cromoli@mpi-hd.mpg.de

\section{Heike Prokoph}

DESY, D-15738 Zeuthen, Germany

E-mail: heike.prokoph@desy.de

\section{for the H.E.S.S. collaboration ${ }^{\dagger}$}

Among the small class of gamma-ray binary systems, PSR B1259-63/LS 2883 is a peculiar case: It is one of two such systems for which the nature of the compact object is known, in this case being a pulsar, and its very eccentric 3.4-year orbit is suitable for frequent observations of periastron passages. Around the times of the periastron passages, bright emission is observed at all wavelengths, enabling detailed studies of particle acceleration and non-thermal photon emission in binary systems.

PSR B1259-63/LS 2883 was observed at very-high-energy (VHE) gamma rays with H.E.S.S. around the times of each periastron periastron passage from 2004 on until 2017. All available data were (re-)analysed using up-to-date analysis tools. Spectra and light curves of this variable gamma-ray emitter are derived from all available data, including phase-folded analysis results. Results from searches for super-orbital variability are presented. Lower limits on spectral cutoff energies are derived, indicating that PSR B1259-63/LS 2883 is an efficient emitter of VHE gamma rays.

In addition, a high-energy (HE) gamma-ray light curve derived from Fermi-LAT measurements is presented. Spectra measured contemporaneously in the HE and VHE gamma-ray domains are compared and discussed in a multi-component context.

36th International Cosmic Ray Conference -ICRC2019-

July 24th - August 1st, 2019

Madison, WI, U.S.A.

\footnotetext{
* Speaker.

${ }^{\dagger}$ for collaboration list see PoS(ICRC2019)1177
} 